\title{
Combined Positioning Method with Single Doppler Radar and Single Optical Theodolite
}

\author{
GONG Zhi-hua ${ }^{1, a^{*}}$, DUAN Peng-wei ${ }^{1, b}$, CHEN Chun-jiang ${ }^{1, c}$, LV Hai-dong ${ }^{1, d}$ \\ Baicheng Ordnance Test Center of China, Baicheng 137001, Jilin \\ email: gzh63298@126.com
}

\begin{abstract}
Keywords: Doppler radar; Optical theodolite; Radial velocity; Function fitting; Hermite function; Least squares
\end{abstract}

Abstract. To make effective use of high-quality radial velocity measured by Doppler radar and improve the positioning accuracy of target trajectory, a combined positioning method with single Doppler radar and single optical theodolite is proposed which is based on target trajectory expressed by function, called function restrict $V A E$ positioning method. Through simulation, comparative calculation with optical intersection positioning method and function restrict $V A E$ positioning is carried out. The results show that, function restrict $V A E$ positioning method takes greater advantage in positioning accuracy, and its requirements for initial value accuracy are low. Therefore, this method is suitable for engineering application, and it is beneficial supplement to tracking and positioning method of moving target.

\section{Introduction}

In the tracking and positioning measurement of target launched by weapons system, all kinds of different measurement equipments play important role of different degree, such as optical theodolite, photo theodolite, pulse radar, continuous wave Doppler radar, phased array radar, space telemetry receiving station and so on. These equipments use unique method for calculating the target location, such as optical intersection algorithm in different planes ${ }^{[1]}$, RAE positioning algorithm with pulse radar and 6V positioning algorithm with Doppler radar ${ }^{[2]}$.Besides, at every sampling time, radial velocity is not independent of other current position parameters, and it will bring certain complexity to calculation, so radial velocity is not taken seriously or used in positioning. But radial velocity is main source of moving object measurement, and it is very important to obtain aerodynamic parameters of moving target, calculate the firing table and evaluate the performance of weapon systems. However, the measurement precision of radial velocity is very high due to the velocity measurement principle of continuous wave radar and the theoretical ultimate precision analysis ${ }^{[3]}$, so moving object positioning by radial velocity is very valuable. Therefore, combined positioning method with radial velocity of single Doppler radar and elevation and azimuth of single optical theodolite is proposed in the paper, called function restrict $V A E$ positioning method. Then simulation analysis of this method is carried out.

\section{Positioning Calculation Model}

Above all, positioning parameters $(x, z, y)$ of moving object should be fitted by function. Fitted function should approximate long trajectory more accurately, that means smaller truncation error. So Hermite function is selected to express positioning parameters of target. The definition of Hermite function is as follow: if function $H(x)$ is $y_{0}, y_{1}, \mathrm{~L}, y_{n-1}$ at $n$ nodes $x_{0}<x_{1}<\mathrm{L}<x_{n-1}$, and 
its values of first derivative is $y_{0}^{\prime}, y_{1}^{\prime}, \mathrm{L}, y_{n-1}^{\prime}$, then $H(x)$ can be approximately replaced by Hermite function as Eq.1.

$$
H(x)=P_{2 n-1}(x)=\sum_{k=0}^{n-1}\left[y_{k}+\left(x-x_{k}\right)\left(y_{k}^{\prime}-2 y_{k} l_{k}^{\prime}(x)\right)\right] l_{k}^{2}(x),
$$

In Eq.1, $\quad l_{k}(x)=\prod_{\substack{j=0 \\ j \neq k}}^{n-1}\left[\frac{\left(x-x_{j}\right)}{\left(x_{k}-x_{j}\right)}\right], \quad l_{k}^{\prime}(x)=\sum_{\substack{j=0 \\ j \neq k}}^{n-1}\left[\frac{1}{x_{k}-x_{j}}\right]$.

Then positioning parameters of target can be expressed by Hermite function as Eq.2.

$$
\left\{\begin{array}{l}
x_{t_{i}}=f_{3}\left(t_{i}, \beta_{x}, T_{x}\right)=\sum_{j=0}^{m_{x}}\left(\beta_{x(j \times 2)} f_{x 1}\left(T_{x j}\right)+\beta_{x(j \times 2+1)} f_{x 2}\left(T_{x j}\right)+\beta_{x(j \times 2+2)} f_{x 3}\left(T_{x j}\right)+\beta_{x(j \times 2+3)} f_{x 4}\left(T_{x j}\right)\right), \\
z_{t_{i}}=f_{3}\left(t_{i}, \beta_{z}, T_{z}\right)=\sum_{j=0}^{m_{\xi}}\left(\beta_{z(j \times 2)} f_{z 1}\left(T_{z j}\right)+\beta_{z(j \times 2+1)} f_{z 2}\left(T_{z j}\right)+\beta_{z(j \times 2+2)} f_{z 3}\left(T_{z j}\right)+\beta_{z(j \times 2+3)} f_{z 4}\left(T_{z j}\right)\right), \\
y_{t_{i}}=f_{3}\left(t_{i}, \beta_{y}, T_{y}\right)=\sum_{j=0}^{m_{y}}\left(\beta_{y(j \times 2)} f_{y 1}\left(T_{y j}\right)+\beta_{y(j \times 2+1)} f_{y 2}\left(T_{y j}\right)+\beta_{y(j \times 2+2)} f_{y 3}\left(T_{y j}\right)+\beta_{y(j \times 2+3)} f_{y 4}\left(T_{y j}\right)\right),
\end{array}\right.
$$

The derivation of Eq.2 is shown as Eq.3.

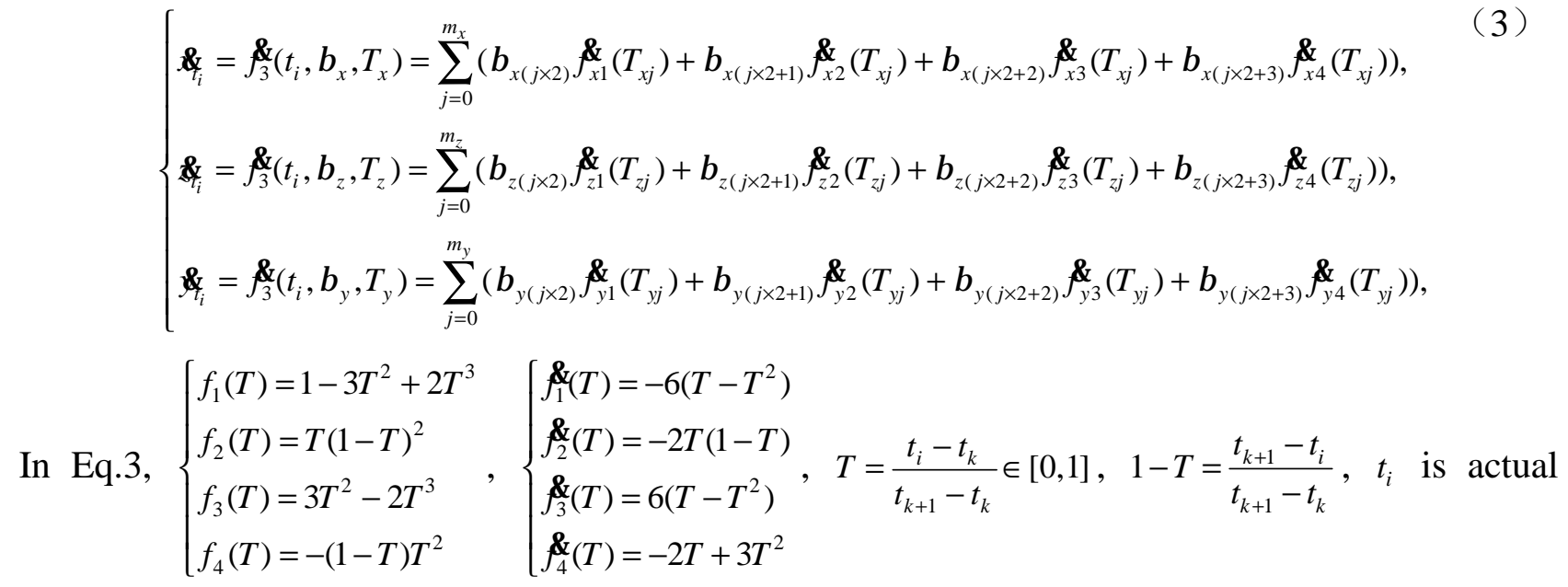
sampling time, $t_{k} \leq t_{i}<t_{k+1}, t_{k} \in T \in\left(T_{x}, T_{z}, T_{y}\right)$, and time is normalized between adjacent nodes. $\left(T_{x}, T_{z}, T_{y}\right),\left(m_{x}, m_{z}, m_{y}\right)$ and $\left(\beta_{x}, \beta_{z}, \beta_{y}\right)$ are fitting function node distribution, internal nodes number and fitting function coefficient to be estimated of target position parameter.

It is nonlinear relationship between target position parameter and the measurement elements, such as radial velocity, azimuth and elevation, so radial velocity, azimuth and elevation can be expressed by basis function as Eq.1 and Eq.2. Then these measurement elements are expand in Taylor series into linear equation, which form combined equation as Eq.4.

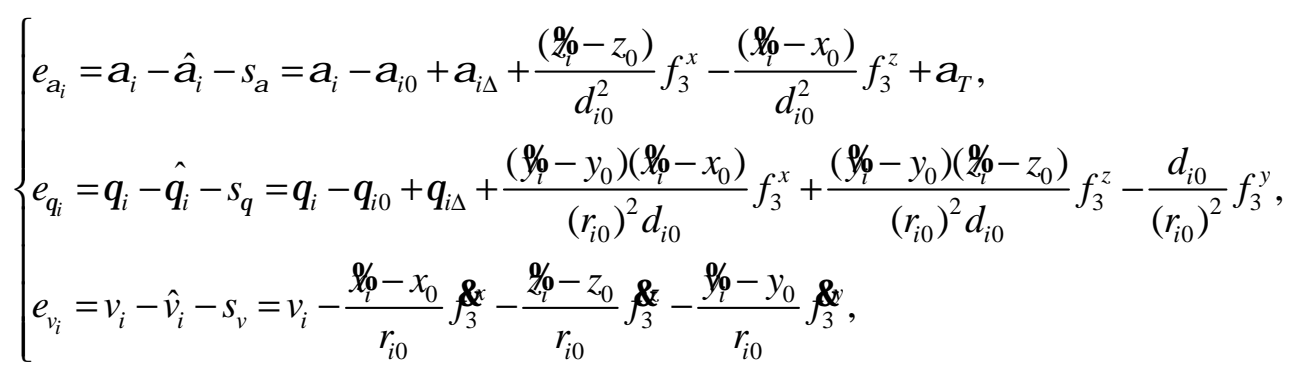


In Eq.4, $f_{3}\left(t_{i}, \beta_{(x, z, y)}, T_{(x, z, y)}\right)$ and $f_{3}^{(x}\left(t_{i}, \beta_{(x, z, y)}, T_{(x, z, y)}\right) f_{3}^{(x, z, y)}$ are replaced by $f_{3}^{\left.\alpha_{x}, z, y\right)}$ and $f_{3}^{\alpha(x, z, y)}$. $\left(\alpha_{i}, \theta_{i}, v_{i}\right)$ is measured data of radial velocity, azimuth and elevation. $\left(\ell_{i} 9 q_{i} 9 g_{i}\right)$. is initial value. $\left(x_{0}, z_{0}, y_{0}\right)$ is measurement station coordinate. $\alpha_{T}$ is judge angle.

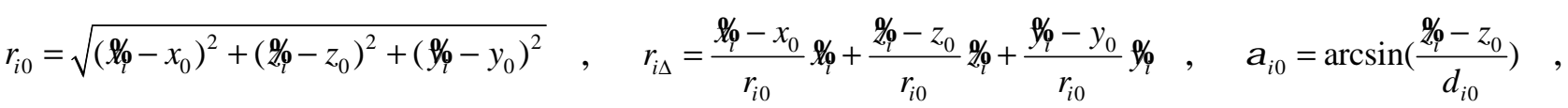

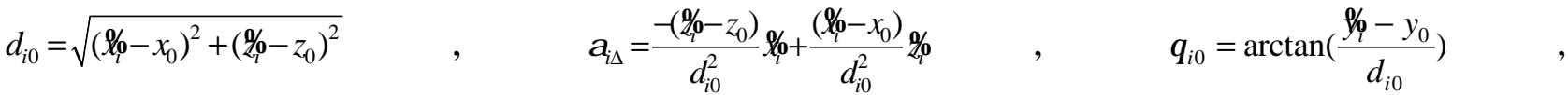

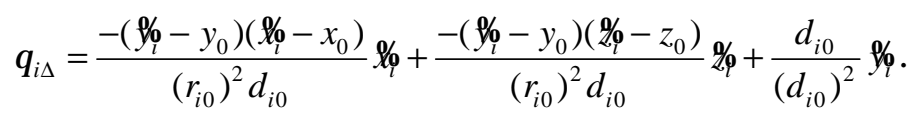

Eq.4 can be changed into matrix form, shown as Eq.5.

$$
\xi=H X+\eta
$$

In Eq.5, error vector $\xi$ is formed by three measurement elements residuals $\left(e_{\alpha}, e_{\theta}, e_{v}\right) . H$ is design matrix for expressing every measurement elements by fitting coefficients of Hermite function $\left(\beta_{x}, \beta_{z}, \beta_{y}\right)$. Parameter vector to be estimated $X$ is formed by fitting coefficients of Hermite function $\left(\beta_{x}, \beta_{z}, \beta_{y}\right) . \eta$ is constant vector of every measurement element.

On the premise that the nodes distribution of Hermite fitting function is known, parameter to be estimated $\left(\beta_{x}, \beta_{z}, \beta_{y}\right)$ and its covariance matrix can be calculated from Eq.5 according to The principle of least squares. And it is shown as Eq.6

$$
\left\{\begin{array}{l}
X=-\left(A^{\mathrm{T}} P A\right)^{-1} A^{\mathrm{T}} P L, \\
\operatorname{Cov}(X)=A^{\mathrm{T}} P A, \\
P=\operatorname{diag}\left(P_{\alpha}, P_{\theta}, P_{v}\right),
\end{array}\right.
$$

In Eq.6, $P$ is weight matrix which is a diagonal matrix formed by statistical random error of measurement elements.

In the process of solution, both the nonlinear function series expansion and approximation of trajectory initial parameters bring truncation errors, so iterative calculation is needed in Eq. 9. Then Hermite function fitting coefficients is substituted into Eq. 2, and the target position parameters are calculated.

From the above positioning method analysis, it is extensible of combined error equation. So more measurement elements can be added to calculate target position. This is function restricted data fusion method.

\section{Simulation and Verification}

First of all, measured data of a Doppler radar and two optical theodolites are simulated. In the launch coordinate system, based on equation of particle motion, a theoretical trajectory and its parameters are calculated. And then, a radial velocity measurement element of Doppler radar and four angle measurement elements of two optical theodolites can be calculated with the theoretical trajectory. According to the engineering application experience, reasonable random error and 
system error are added into these five simulated measurement elements which is shown as Tab.1. In the simulation, Doppler radar is placed at rear side of the launch site, and two optical theodolites are placed at left side of the launch site. The theoretical trajectory is independently added fixed errors $(800 \mathrm{~m}, 1000 \mathrm{~m}, 700 \mathrm{~m})$ and random errors $(6 \mathrm{~m}, 6 \mathrm{~m}, 8 \mathrm{~m})$ as initial value of $(x, z, y)$.

\begin{tabular}{|c|c|c|c|c|c|}
\hline \multirow{2}{*}{$\begin{array}{c}\text { Measuring } \\
\text { equipments } \\
\text { Measuring } \\
\text { elements }\end{array}$} & \multicolumn{2}{|c|}{$\begin{array}{c}\text { Theodolite } 1 \# \\
\text { station }\end{array}$} & \multicolumn{2}{|c|}{$\begin{array}{c}\text { Theodolite } 2 \# \\
\text { station }\end{array}$} & \multirow{2}{*}{$\begin{array}{c}\text { Continuous-wave } \\
\text { radar }\end{array}$} \\
\hline & $\mathrm{A} / \mathrm{sec}$ & $\mathrm{E} / \mathrm{sec}$ & $\mathrm{A} / \mathrm{sec}$ & $\mathrm{E} / \mathrm{sec}$ & \\
\hline $\begin{array}{c}\text { Random error } \\
\text { (True) }\end{array}$ & 20 & 20 & 20 & 20 & 0.30 \\
\hline $\begin{array}{c}\text { System error } \\
\text { (True)) }\end{array}$ & 5 & -10 & -10 & -10 & 0.00 \\
\hline
\end{tabular}

\section{Determination of Hermite function nodes}

As the above analysis, in actual calculation, Hermite function nodes distribution must be determined first when target positioning parameters are expressed by Hermite function. For node selection, the first idea is equal interval nodes. Its essence is using a constant frequency bandwidth filter for data filtering. However, because there is much noise in frequency the non-stationary data and there is special point, so when using a constant frequency bandwidth filter for data filtering, it must increase the nodes density to increase the filter bandwidth [6], achieve more accurate and reduce the truncation error. This leads to a surge in parameters to be estimated and militates against solution of equation. Consequently, it must use non free node distribution. To get appropriate distribution, it can be selected according to the acceleration change characteristics of target trajectory [7]. When the target's acceleration changes violently, dense nodes distribution is used, but the target's acceleration changes smoothly, sparse nodes distribution is used.

The target's acceleration can be calculated by simulated radial velocity of Doppler radar with three order central smoothing computation, which is shown as Fig.1.

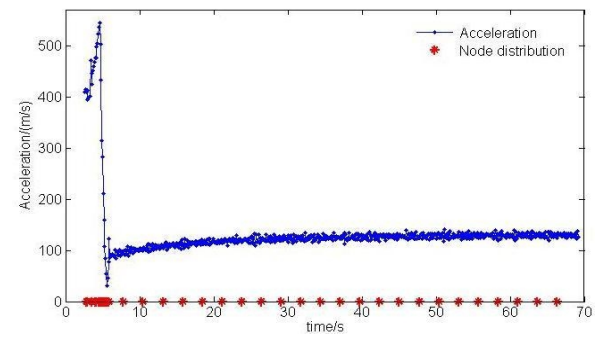

Fig.1 Free nodes selection of function based on acceleration character of moving target

From Fig.1, in the first seven seconds period, the target's acceleration change violently, so dense nodes distribution with more than ten nodes is used. In the later more tan sixty seconds period, the acceleration change smoothly, so sparse nodes distribution with only about twenty nodes is used. This insures that the positioning parameters of target are accurately expressed by Hermite function, and reasonably save the number of parameters to be estimated.

\section{Positioning error analysis}

Based on determining the nodes distribution of Hermite function, simulated data is calculated by function restrict $V A E$ positioning method. To verify the effect of the calculating method, this method is compared with optical nonplanar intersection method. First, combined positioning is calculated with radial velocity of Doppler radar and azimuth and elevation of theodolite 1 or theodolite 2. And the positioning error can be calculated with theoretical target trajectory. Secondly, optical nonplanar 
intersection is computed with simulated data of two theodolites. The positioning error also can be calculated with theoretical target trajectory. Last, statistical error of these two methods is compared to examine the positioning effect of the new method. Through calculation, positioning error curves of these two methods are shown as Fig.2 and their statistical error is shown as Tab.1.
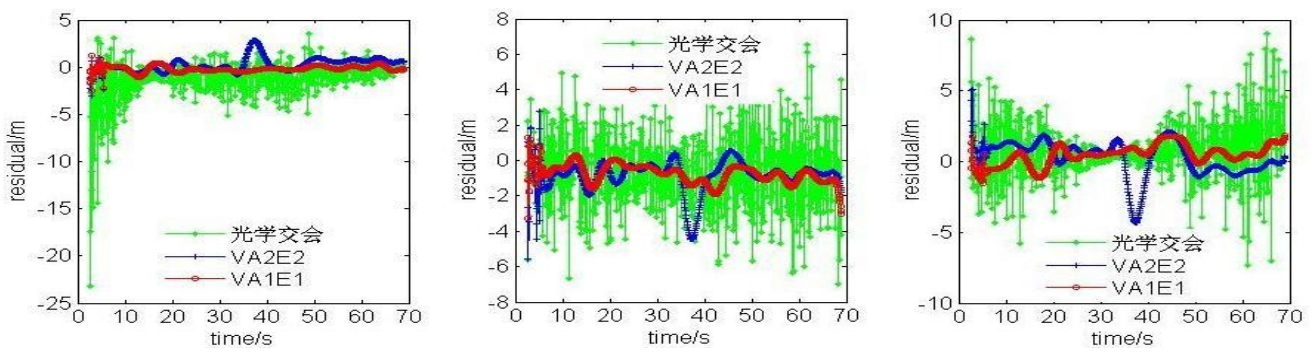

Fig.2 Error caves of two positioning methods

Tab.2 Error statistics of two positioning methods

\begin{tabular}{cccccc}
\hline Positioning method & Statistic parameter & $\mathrm{x} / \mathrm{m}$ & $\mathrm{z} / \mathrm{m}$ & $\mathrm{y} / \mathrm{m}$ & $\sigma / \mathrm{m}$ \\
\hline \multirow{3}{*}{ Optical intersection } & Mean & -1.264 & -0.669 & 0.729 & 1.605 \\
& Mean-square deviation & 2.179 & 2.111 & 1.993 & 3.630 \\
& Total error & 2.519 & 2.214 & 2.122 & 3.969 \\
\hline \multirow{2}{*}{ Function restricted $V A E$} & Mean & -0.182 & -0.758 & 0.481 & 0.916 \\
$(1 \#)$ & Mean-square deviation & 0.335 & 0.542 & 0.712 & 0.955 \\
& Total error & 0.318 & 0.932 & 0.859 & 1.307 \\
\hline \multirow{2}{*}{ Function restricted $V A E$} & Mean & 0.262 & -0.835 & 0.277 & 0.918 \\
$(2 \#)$ & Mean-square deviation & 0.745 & 0.944 & 1.280 & 1.756 \\
& Total error & 0.790 & 1.260 & 1.310 & 1.982 \\
\hline
\end{tabular}

From Fig.2 and Tab.2, for the simulated data, the positioning precision of function restrict VAE method with not only Doppler radar and theodolite 1 but also Doppler radar and theodolite 2 is at least one time higher than optical nonplanar intersection method. And this effectively confirms the correctness and practicability of the new positioning method. However, in combined positioning with Doppler radar and theodolite 2, there is a error peak at about 40 seconds. Why? Whether it is relative to the space geometry relation of the equipment location and trajectory or not, it needs further verification. And in this paper, we do not study it.

\section{Conclusion}

Based on the calculation of above simulation and verification, we can draw the following conclusions.

Based on the idea of expressing the trajectory with function, Doppler radar and optical theodolite which are not related are combined, and a function restrict VAE positioning method is established with radial velocity of Doppler radar and measuring angle of single optical theodolite. It is verified that this method has higher positioning precision, better stability of computation and its initial value does not requires high accuracy. It is suitable for engineering application and it is a useful complement to tracking algorithm for moving target positioning.

Under the condition of maximum reducing the measurement elements for positioning, function restrict $V A E$ method effectively uses the high precision radial velocity of Doppler radar. This method decreases the system error of positioning data and effectively suppresses the random error of positioning data that is the key reason why this method has higher positioning precision than optical intersection positioning method at present.

Based on the positioning calculation model of function restrict VAE method, measurement elements involved in the calculation of various types equipments can be unlimited expanded. This is 
helpful for increasing the positioning precision of target and effective for calibrating the system error of every measurement element, but it may decrease the computational efficiency.

Considering the limitations of the designed simulation data, in practical engineering application, some problems still need further research and explore, such as whether this method is affected by the geometric relationship among the equipment station.

\section{Reference}

[1] Hou Hong-lu, LI Hong. Measurement of vehicle three dimension coordinate with photoelectrical theodolite and error analysis[J]. Opto-Electronic Engineering June, 2002, 29 (3): 4-8. (in Chinese)

[2] Jia Xing-quan.Continuous-wave radar data processing. Beijing: National Defensen Industry Press, 2005. (in Chinese)

[3] Gong Zhi-hua, Duan Peng-wei, Dong Li-tao. Ultimate precision analysis and simulation of trajectory measurement radar[J]. Modern Radar, 2012，34(6):74-77. (in Chinese)

[4] ZHAN Wu-ping, SHEN Ting-zheng, LIU Cheng, LIU Qiao-ling. Hermite interpolation fusion method for netted radar measuring colored noise data[J]. Modern Radar, 2012, 34(6): 41-44. (in Chinese)

[5] XU Shi-liang. Commonly used algorithm for assembly (C language description) [M]. Beijing: Tsinghua University Press, 2004.

[6] Gong Zhi-hua,Zhou Hai-yin,Guo Wen-sheng,XU Xu.Data fusion algorithm for target trajectory determination based on spline function representation[J].ACTA ARMAMENTARII,2014,35(1):120-127.( in Chinese) [7] Zhu Ju-bo, The incompletely measured data modeling and appling. Graduate school nation university of defense technology, May 2004. 